\title{
Studying Ideal Semantics via Logic Programming Semantics
}

\author{
Juan Carlos Nieves \\ Universitat Politècnica de Catalunya \\ Departament de Lleguatges i Sistemes Informàtics \\ c/Jordi Girona 1-3, E08034, Barcelona, Spain \\ Email: jcnieves@lsi.upc.edu
}

\author{
Mauricio Osorio \\ Universidad de las Américas - Puebla \\ Depto. de Actuaría, Física y Matemáticas \\ Sta. Catarina Mártir, Cholula, Puebla, 72820 México \\ Email:osoriomauri@googlemail.com
}

\begin{abstract}
In this paper, we show that by using extensions of the Well-Founded Semantics (WFS) which were defined in terms of rewriting systems, one can characterize ideal sets. We also show that these extensions of the well-founded semantics define argumentation semantics with similar behaviour to the ideal argumentation semantics. On the other hand, we introduce a new logic programming semantics which is able to characterize the ideal sets of an argumentation framework.
\end{abstract}

Keywords-Abstract Argumentation Semantics, Logic Programming Semantics, Non-monotonic Reasoning.

\section{INTRODUCTION}

Although several approaches have been proposed for argument theory, Dung's approach presented in [8], is a unifying framework which has played an influential role on argumentation research and Artificial Intelligence (AI). Dung's approach is regarded as an abstract model where the main concern is to find the set of arguments which are considered as acceptable. The strategy for inferring the set of acceptable arguments is based on abstract argumentation semantics.

The kernel of Dung's framework is supported by four abstract argumentation semantics: stable semantics, preferred semantics, grounded semantics, and complete semantics. Even thought each of these argumentation semantics represents different patterns of selection of arguments, all of them are based on the basic concept of admissible sets. Informally speaking, an admissible set represents a coherent and defendable point of view in a conflict between arguments. The stable, preferred and complete semantics represent a credulous reasoning approach. This means that for a given problem these semantics identify a set of possible solutions. Unlike the stable, preferred and complete semantics, the grounded semantics represents a sceptical reasoning approach. One of the main features of the grounded semantics is that it is polynomial time computable. This fact has encouraged to use the grounded semantics in real argumentation-based systems [4], [12]. However, a common problem of the grounded semantics is that it is easy to identify a set of argumentation frameworks in which the grounded semantics is empty. Due to this weakness of the grounded semantics, Dung et al. [9] have introduced an extension of the grounded semantics which is called ideal semantics. Due to the sceptical reasoning properties which satisfy the ideal semantics [1], the ideal semantics has been explored from different points of view [1], [9], [10]. Like the stable, preferred, grounded, complete semantics, the ideal semantics is based on the concept of admissible set.

Since Dung's approach was introduced in [8], it was viewed as a special form of logic programming with negation as failure. For instance, in [8] it was proved that the grounded semantics can be characterized by the wellfounded semantics (WFS) [13]. Given that the ideal semantics is an extension of the grounded semantics, one can expect that the ideal semantics can be studied by extensions of the well-founded semantics.

One can identify at least two approaches for exploring argumentation semantics from the logic programming perspective. On the one hand, one can find some approaches which are mainly concerned in the operational objectives of the argumentation semantics. This means that the main concern of these approaches is to identify an efficient implementation of the given argumentation semantics [11], [16]. These approaches are mainly based on only one logic programming semantics and different codifications of the argumentation semantics. On the other hand, one can find other approaches which characterize different argumentation semantics by considering a fixed logic programming specification of an argumentation framework and different logic programming semantics [3], [8]. The last approach possibly is the more adequate approach for exploring the common non-monotonic reasoning properties between argumentation semantics and logic programming semantics than the former one. This observation is based on the fact that one can identify the set of acceptable arguments of a given argumentation framework by considering only the models of a particular logic program.

In this paper, we extended the results of [3], [8] in which the authors consider a fixed logic programming specification of an argumentation framework and different logic programming semantics for capturing different selection-patterns of arguments from an argumentation framework. In particular, we explore some extensions of the well-founded semantics which are able to characterize ideal sets. Moreover, we introduce a new logic programming semantics which is able 
to characterize the ideal sets of an argumentation framework. For constructing this new logic programming semantics, the p-stable semantics is considered [15]. It is worth mentioning that the p-stable semantics is a logic programming semantics able to characterize the preferred argumentation semantics [3].

Since the ideal sets are characterized by logic programming semantics such WFS and $\mathrm{WFS}^{+}$which are wellbehaved semantics in logic programming [6], the results presented in this paper suggest that:

- the ideal semantics satisfies some expected nonmonotonic reasoning properties.

- the less restricted scepticism of the ideal semantics is supported by reasoning inferences such as reasoning by cases and logic consequence.

The rest of the paper is divided as follows: in order to have a self contained document all the necessary background is introduced in $\S$ II. In $\S$ III, a transformation of an argumentation framework into a normal logic program is presented. In $\S I V$, the main results of this paper are presented. Finally in the last section, our conclusions and future work is outlined.

\section{BACKGROUND}

In this section, we first define the syntax of a valid logic program, after that some extensions of the Well-Founded Semantics and the p-stable semantics are introduced. In the last part of this section, we present some basic concepts of argumentation theory.

\section{A. Logic programs: Syntax}

A signature $\mathcal{L}$ is a finite set of elements that we call atoms. A literal is an atom, $a$, or the negation of an atom not $a$. Given a set of atoms $\left\{a_{1}, \ldots, a_{n}\right\}$, we write not $\left\{a_{1}, \ldots, a_{n}\right\}$ to denote the set of literals \{not $a_{1}, \ldots$, not $\left.a_{n}\right\}$. A normal clause is of the form: $a_{0} \leftarrow a_{1}, \ldots, a_{j}$, not $a_{j+1}, \ldots$, not $a_{n}$, where $a_{i}$ is an atom, $0 \leq i \leq n$. When $n=0$ the normal clause is an abbreviation of $a_{0} \leftarrow \top$, where $\top$ is the ever true atom. A normal program is a finite set of normal clauses. Sometimes, we denote a clause $C$ by $a \leftarrow \mathcal{B}^{+}, \neg \mathcal{B}^{-}$, where $\mathcal{B}^{+}$contains all the positive body literals and $\mathcal{B}^{-}$contains all the negative body literals. We also use $\operatorname{body}(C)$ to denote $\mathcal{B}^{+}, \neg \mathcal{B}^{-}$. When $\mathcal{B}^{-}=\emptyset$, the clause $C$ is called definite clause. A definite program is a finite set of definite clauses. We denote by $\mathcal{L}_{P}$ the signature of $P$, i.e. the set of atoms that occurs in P. Given a signature $\mathcal{L}$, we write $\operatorname{Prog}_{\mathcal{L}}$ to denote the set of all the programs defined over $\mathcal{L}$.

\section{B. P-stable semantics}

Logic consequence in classic logic is denoted by $\vdash$. Given a set of proposition symbols $S$ and a theory (a set of wellformed formulae) $\Gamma$, if $\Gamma \vdash S$ if and only if $\forall s \in S \Gamma \vdash s$. When we treat a logic program as a theory, each negative literal not $a$ is replaced by $\sim a$ such that $\sim$ is regarded as the classical negation in classic logic. Given a normal program $\mathrm{P}$, if $M \subseteq \mathcal{L}_{P}$, we write $P \Vdash M$ when: $P \vdash M$ and $M$ is a classical 2-valued model of $P$ (i.e. atoms in $M$ are set to true, and atoms not in $M$ are set to false; the set of atoms is a classical model of $P$ if the induced interpretation evaluates $P$ to true).

$\mathrm{P}$-stable semantics is defined in terms of a single reduction which is defined as follows:

Definition 1: [15] Let $P$ be a normal program and $\mathrm{M}$ be a set of literals. We define $\operatorname{RED}(P, M):=\{l \leftarrow$ $\left.\mathcal{B}^{+}, \operatorname{not}\left(\mathcal{B}^{-} \cap M\right) \mid l \leftarrow \mathcal{B}^{+}, \operatorname{not} \mathcal{B}^{-} \in P\right\}$.

Let us consider the set of atoms $M_{1}:=\{a, b\}$ and the following normal program $P_{1}: a \leftarrow \operatorname{not} b$, not $c$.

$a \leftarrow b . \quad b \leftarrow a$.

We can see that $\operatorname{RED}\left(P_{1}, M_{1}\right)$ is: $a \leftarrow$ not $b . \quad a \leftarrow b$. $b \leftarrow a$.

By considering the reduction $R E D$, the $p$-stable semantics for normal programs is defined.

Definition 2: [15] Let $P$ be a normal program and $M$ be a set of atoms. We say that $M$ is a p-stable model of $P$ if $R E D(P, M) \Vdash M$. Pstable $(P)$ denotes the set of p-stable models of $P$.

Let us consider again $M_{1}$ and $P_{1}$ in order to illustrate the definition. We want to verify whether $M_{1}$ is a p-stable model of $P_{1}$. First, we can see that $M_{1}$ is a model of $P_{1}$, i.e. $\forall C \in P_{1}, M_{1}$ evaluates $C$ to true. Moreover, we can see that each atom of $M_{1}$ can be proved from $\operatorname{RED}\left(P_{1}, M_{1}\right)$ by using classical inference. Then we can conclude that $R E D\left(P_{1}, M_{1}\right) \Vdash M_{1}$. Hence, $M_{1}$ is a $p$-stable model of $P_{1}$.

\section{The Well-Founded Semantics and extensions}

First of all, we present some definitions w.r.t. 3-valued logic semantics.

A partial interpretation based on a signature $\mathcal{L}$ is a disjoint pair of sets $\left\langle I_{1}, I_{2}\right\rangle$ such that $I_{1} \cup I_{2} \subseteq \mathcal{L}$. A partial interpretation is total if $I_{1} \cup I_{2}=\mathcal{L}$. Given two interpretations $I=\left\langle I_{1}, I_{2}\right\rangle, J=\left\langle J_{1}, J_{2}\right\rangle$, we set $I \leq_{k} J$ if, by definition, $I_{i} \subseteq J_{i}, i=1,2$. Clearly $\leq_{k}$ is a partial order. When we look at interpretations as sets of literals then $\leq_{k}$ corresponds to $\subseteq$. A general semantics SEM is a function on $\operatorname{Prog}_{\mathcal{L}}$ which associates with every program a partial interpretation.

Given a signature $\mathcal{L}$ and two semantics $\mathrm{SEM}_{1}$ and $\mathrm{SEM}_{2}$, we define $\mathrm{SEM}_{1} \leq_{k} \mathrm{SEM}_{2}$ if for every program $P \in \operatorname{Prog}_{\mathcal{L}}$, $\operatorname{SEM}_{1}(P) \leq_{k} \operatorname{SEM}_{2}(P)$.

Definition 3 (SEM): For any logic program $P$, we define $\operatorname{HEAD}(P)=\left\{a \mid a \leftarrow \mathcal{B}^{+}, \neg \mathcal{B}^{-} \in P\right\}-$ the set of all head-atoms of $P$. We also define $\operatorname{SEM}(P)=$ $\left\langle P^{\text {true }}, P^{\text {false }}\right\rangle$, where $P^{\text {true }}:=\{p \mid p \leftarrow \top \in P\}$ and $P^{\text {false }}:=\left\{p \mid p \in \mathcal{L}_{P} \backslash \operatorname{HEAD}(P)\right\}$.

Now, we define some basic transformation rules for normal logic programs which will be considered for characterizing WFS. 
Definition 4 (Basic Transformation Rules): [7] A transformation rule is a binary relation on $\operatorname{Prog}_{\mathcal{L}}$. The following transformation rules are called basic. Let a program $P \in \operatorname{Prog}_{\mathcal{L}}$ be given.

$\mathrm{RED}^{+}$:This transformation can be applied to $P$, if there is an atom $a$ which does not occur in $\operatorname{HEAD}(\mathrm{P})$. RED $^{+}$transforms $P$ to the program where all occurrences of not $a$ are removed.

$\mathrm{RED}^{-}$:This transformation can be applied to $P$, if there is a rule $a \leftarrow \top \in P$. RED ${ }^{-}$transforms $P$ to the program where all clauses that contain not $a$ in their bodies are deleted.

Succ: Suppose that $P$ includes a fact $a \leftarrow \top$ and a clause $q \leftarrow \operatorname{body}$ such that $a \in \operatorname{bod} y$. Then we replace the clause $q \leftarrow$ body by $q \leftarrow \operatorname{bod} y \backslash\{a\}$.

FailureSuppose that $P$ contains a clause $q \leftarrow$ body such that $a \in b o d y$ and $a \notin H E A D(P)$. Then we erase the given clause.

Loop: We say that $P_{2}$ results from $P_{1}$ by $\operatorname{Loop}_{A}$ if, by definition, there is a set $A$ of atoms such that: 1 . for each rule $a \leftarrow$ body $\in P_{1}$, if $a \in A$, then body $\cap$ $A \neq \emptyset$, 2. $P_{2}:=\left\{a \leftarrow \operatorname{bod} y \in P_{1} \mid \operatorname{bod} y \cap A=\emptyset\right\}$, 3. $P_{1} \neq P_{2}$.

SUB : If $P$ contains two clauses $a \leftarrow \operatorname{bod} y_{1}$ and $a \leftarrow$ $\operatorname{bod}_{2}$, where body $\subseteq \operatorname{bod}_{2}$, then $a \leftarrow \operatorname{body}_{2}$ is removed from $P$

TAUT Suppose $P$ contains a rule $C$ which has the same atom in its head and its positive body. Then we remove this rule.

LC : Suppose $P \vdash a$ for an atom $a$. Then we add the rule $a \leftarrow \top$ in $P$.

Let $C S_{0}$ be the rewriting system such that contains the transformation rules: $R E D^{+}, R E D^{-}$, Succ, Failure, and Loop and $C S_{1}=C S_{0} \cup\{S U B, T A U T, L C\}$.

We denote the uniquely determined normal form of a program $P$ with respect to the system $\mathcal{C S}$ by $\operatorname{norm}_{\mathcal{C S}}(P)$. Every system $\mathcal{C S}$ induces a semantics $\mathrm{SEM}_{\mathcal{C S}}$ as follows: $\operatorname{SEM}_{\mathcal{C S}}(P):=\operatorname{SEM}\left(\right.$ norm $\left._{\mathcal{C S}}(P)\right)$. In order to illustrate the basic transformation rules, let us consider the following example.

Example 1: Let $P$ be the following normal program: $\operatorname{def}(b) \leftarrow \operatorname{not} \operatorname{def}(a) . \quad \operatorname{def}(b) \leftarrow \top$.

$\operatorname{def}(c) \leftarrow \operatorname{def}(a) . \quad \operatorname{def}(c) \leftarrow \operatorname{not} \operatorname{def}(b)$.

Now, let us apply $C S_{0}$ to $P$. Since $\operatorname{def}(a) \notin H E A D(P)$, then, we can apply $\mathbf{R E D}^{+}$to $P$. Thus we get: $\operatorname{def}(b) \leftarrow \top$. $\operatorname{def}(c) \leftarrow \operatorname{not} \operatorname{def}(b) . \quad \operatorname{def}(c) \leftarrow \operatorname{def}(a)$.

Notice that we can apply $\mathbf{R E D}^{-}$to the new program, thus we get: $\operatorname{def}(b) \leftarrow \top . \quad \operatorname{def}(c) \leftarrow \operatorname{def}(a)$.

Finally, we can apply Failure to the new program, thus we get: $\operatorname{def}(b) \leftarrow \top$. This last program is the normal form of $P$ w.r.t. $C S_{0}$, because none of the transformation rules from $C S_{0}$ can be applied.

WFS is one of the most acceptable semantics in logic programming. It was introduced in [13] and was characterized in terms of rewriting systems in [2]. This characterization is defined as follows:

Theorem 1: [2] $C S_{0}$ is a confluent rewriting system. It induces a 3-valued semantics that it is the Well-founded Semantics.

There is an extension of WFS which is called $W F S^{+}$, this extension of WFS is a logic programming semantics which like WFS is a well-behaved semantics [6]. WFS was introduced in [5] and characterized in terms of rewriting systems in [7].

Theorem 2: [7] The $W F S^{+}$semantics is induced by the confluent rewriting system $C S_{1}$.

The consideration of rewriting systems which are confluent and terminating defines a general methodology for characterizing different logic programming semantics. Observe that the differences between WFS and $\mathrm{WFS}^{+}$are three rewriting rules, i.e. SUB, TAUT, LC. Each rewriting rules defines different levels of inference in a logic programming semantics.

Now we present two other rewriting rules which help to define three extensions of WFS with different levels of inference.

Definition 5: [7]

$L L C^{\prime}$ : Let $a$ be an atom that occurs negatively in a program $P$ and also appears in the head of some rule. Let $P_{1}$ be the program that results from $P$ by removing not a from every clause of $P$. Let Succ* denote the reflexive and transitive closure of the relation Succ. Suppose that $P_{1}$ relates to $P_{2}$ by Succ* and $a \in P_{2}$. In this case, we add $a \leftarrow \top$ to $P$.

WK: Let $P$ be a program and suppose the following condition holds: $C_{1} \in P, C_{2} \in P, C_{1}$ is of the form $a \leftarrow l$ and $C_{2}$ is of the form $a \leftarrow$ not $l$. Then the $W K$ transformation replaces the clauses $C_{1}$ and $C_{2}$ in $P$ by the single clause $a \leftarrow \top$.

By considering $L L C^{\prime}$ and $W K$, one can define three extensions of WFS as follows:

Lemma 1: [7] Let $C S_{2}:=C S_{0} \cup\left\{L L C^{\prime}\right\}, C S_{3}:=$ $C S_{0} \cup\{W K\}$ and $C S_{4}:=C S_{0} \cup\left\{L L C^{\prime}, W K\right\}$.

- $C S_{2}$ is a confluent rewriting system. It induces a 3valued semantics that we call $W F S^{L L C^{\prime}}$.

- $C S_{3}$ is a confluent rewriting system. It induces a 3 valued semantics that we call $W F S^{W K}$.

- $C S_{4}$ is a confluent rewriting system. It induces a 3 valued semantics that we call $W F S^{W K+L L C^{\prime}}$.

\section{Argumentation theory}

Now, we define some basic concepts of Dung's argumentation approach. The first one is an argumentation framework. An argumentation framework captures the relationships between arguments.

Definition 6: [8] An argumentation framework is a pair $A F:=\langle A R$, attacks $\rangle$, where $A R$ is a finite set of arguments, 
and attacks is a binary relation on $A R$, i.e. attacks $\subseteq A R \times$ $A R$.

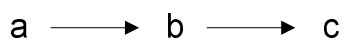

Figure 1. A single argumentation framework

An argumentation framework can be regarded as a directed graph. For instance, if $A F$ := $\langle\{a, b, c\},\{(a, b),(b, c)\}\rangle$, then $A F$ is represented as in Figure 1. We say that $a$ attacks $b$ (or $b$ is attacked by $a$ ) if attacks $(a, b)$ holds. Similarly, we say that a set $S$ of arguments attacks $b$ (or $b$ is attacked by $S$ ) if $b$ is attacked by an argument in $S$.

Definition 7: [8]

- A set $S$ of arguments is said to be conflict-free if there are no arguments $a, b$ in $S$ such that $a$ attacks $b$.

- An argument $a \in A R$ is acceptable with respect to a set $S$ of arguments if and only if for each argument $b \in A R$ : If $b$ attacks $a$ then $b$ is attacked by $S$.

- A conflict-free set of arguments $S$ is admissible if and only if each argument in $S$ is acceptable w.r.t. $S$.

Definition 8: Let $A F:=\langle A R$, attacks $\rangle$ and $S \subseteq A R$. A set of argument $S$ is:

- preferred if and only if $S$ is a maximal (w.r.t. inclusion) admissible set of $A F$ [8].

- ideal if and only if $S$ is admissible and it is contained in every preferred set of $A F$ [9].

\section{MAPPING FROM ARGUMENTATION FRAMEWORKS TO NORMAL PROGRAMS}

In order to see an argumentation framework as a normal program, a mapping from an argumentation framework into a normal logic program is presented. This mapping was introduced in [14]. In this mapping, the predicate $\operatorname{def}(x)$, where the intended meaning of $\operatorname{def}(x)$ is " $x$ is a defeated argument", is used.

Definition 9: Let $A F:=\langle A R$,attacks $\rangle$ be an argumentation framework and $a \in A R$. We define the transformation function $\Psi(a)$ as follows:

$$
\begin{gathered}
\Psi(a)=\bigcup_{b:(b, a) \in \text { attacks }}\{\operatorname{def}(a) \leftarrow \operatorname{not} \operatorname{def}(b)\} \cup \\
\bigcup_{b:(b, a) \in \text { attacks }}\left\{\operatorname{def}(a) \leftarrow \bigwedge_{c:(c, b) \in \text { attacks }} \operatorname{def}(c)\right\}
\end{gathered}
$$

The direct generalization of the transformation function $\Psi$ to an argumentation framework is defined as follows:.

Definition 10: Let $A F:=\langle A R$, Attacks $\rangle$ be an argumentation framework. We define its associated normal program as follows: $\Psi_{A F}:=\bigcup_{a \in A R}\left\{\Psi_{1}(a)\right\}$.

As illustration of this transformation, the normal program $P$ of Example 1 is the associated normal program of the argumentation framework of Figure 1.
Before moving on, we want to observe that by considering $\Psi_{A F}$, the well-founded semantics and the Pstable semantic, one can characterize the grounded and the preferred semantics respectively [3].

\section{Approaching IdEAL SETS}

In this section, the main results of this paper are presented. In particular, a set of characterizations of ideal sets in terms of logic programming semantics are presented. Moreover the concept of ideal model for normal logic programs is defined. As an application of the ideal models, we show that ideal models are able to characterize the ideal set of an argumentation framework.

We start presenting a set of characterizations of the ideal sets which are based on WFS and its extensions (presented in Section II-C). For this setting, the following notation is introduced. Given an argumentation framework $A F:=$ $\langle A R$, attacks $\rangle$ and $S \subseteq A R, f(S)=\{\operatorname{def}(a) \mid a \in S\}$.

Lemma 2: Let $A F:=\langle A R$,attacks $\rangle$ be an argumentation framework and $S, D \subseteq A R$.

1) if $W F S\left(\Psi_{A F}\right)=\langle f(D), f(S)\rangle$, then $S$ is an ideal set, denoted by $I^{W F S}(A F)$.

2) if $W F S^{L L C^{\prime}}\left(\Psi_{A F}\right)=\langle f(D), f(S)\rangle$, then $S$ is an ideal set, denoted by $I^{W F S^{L L C^{\prime}}}(A F)$.

3) if $W F S^{W K}\left(\Psi_{A F}\right)=\langle f(D), f(S)\rangle$, then $S$ is an ideal set, denoted by $I^{W F S^{W K}}(A F)$.

4) if $W F S^{W K+L L C^{\prime}}\left(\Psi_{A F}\right)=\langle f(D), f(S)\rangle$, then $S$ is an ideal set, denoted by $I^{W F S^{W K+L L C^{\prime}}}(A F)$.

5) if $W F S^{+}\left(\Psi_{A F}\right)=\langle f(D), f(S)\rangle$, then $S$ is an ideal set, denoted by $I^{W F S^{+}}(A F)$.

Proof: (sketch) (1) follows by Theorem 7 from [3] which proves that WFS and $\Psi_{A F}$ characterize the grounded extensions and the fact that the grounded extension is an ideal set. The reminder points follows by (1) and the following four observations:

1) The 2-valued models of $\Psi_{A F}$ characterize the admissible sets of $A F$.

2) If $S E M\left(P_{A F}\right)=\langle f(D), f(S)\rangle$ such that $S E M$ is either $W F S, W F S^{L L C^{\prime}}, W F S^{W K}, W F S^{W K+L L C^{\prime}}$ or $W F S^{+}$, then $\mathcal{L}_{P_{A F}} \backslash f(S)$ is a 2-valued model of $\Psi_{A F}$ and $S$ is an admissible se of $A F$.

3) The p-stable models of $\Psi_{A F}$ characterizes the preferred extensions of $A F$.

4) The positive atoms of $W F S, W F S^{L L C^{\prime}}, W F S^{W K}$, $W F S^{W K+L L C^{\prime}}$ and $W F S^{+}$belongs to every pstable model.

Observe that this lemma suggests that the less restricted scepticism of the ideal semantics is supported by reasoning inferences such as reasoning by cases (i.e. WK) and local logic consequence (i.e. LLC). Let us remember that the transformation rule $W S$ captures the idea of a reasoning 
by cases and the transformation rule $L L C$ captures the idea of a reasoning based on Local Logic Consequence.

In order to illustrate these characterizations of ideal sets, let us consider the following examples.

Example 2: Let $A F:=\langle A R$, attacks $\rangle$ be an argumentation framework, in which $A R:=\{a, b, c\}$ and attacks $:=$ $\{(a, a),(a, b),(b, c),(c, b)\}$ (see Figure 2). Hence, $\Psi_{A F}$ is:

$$
\begin{array}{llrl}
\operatorname{def}(a) \leftarrow \operatorname{not} \operatorname{def}(a) . & & \operatorname{def}(a) \leftarrow \operatorname{def}(a) . \\
\operatorname{def}(b) \leftarrow \operatorname{not} \operatorname{def}(a) . & & \operatorname{def}(b) \leftarrow \operatorname{not} \operatorname{def}(c) . \\
\operatorname{def}(b) \leftarrow \operatorname{def}(a) . & & \operatorname{def}(b) \leftarrow \operatorname{def}(c) . \\
\operatorname{def}(c) \leftarrow \operatorname{not} \operatorname{def}(b) . & & \operatorname{def}(c) \leftarrow \operatorname{def}(c), \operatorname{def}(a) .
\end{array}
$$

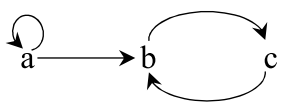

Figure 2. An argumentation framework with two-length cycle and a selfdefeated argument.

We can see that the argumentation framework $A F$ has a preferred extension which is $\{c\}$ and the grounded extension is empty. It is obvious that this argumentation framework has two ideal sets: \{\} and $\{c\}$. Now let us see which idea sets are inferred by the argumentation semantics introduced in Lemma 2.

\begin{tabular}{|l|c|c|}
\hline Semantics & 3-logic programming model & Ideal Set \\
\hline$W F S\left(\Psi_{A F}\right)$ & $\langle\{\},\{\}\rangle$ & \{\} \\
\hline$W F S^{L L C^{\prime}}\left(\Psi_{A F}\right)$ & $\langle\{\operatorname{def}(a), \operatorname{def}(b)\},\{\operatorname{def}(c)\}\rangle$ & $\{c\}$ \\
\hline$W F S^{W K}\left(\Psi_{A F}\right)$ & $\langle\{\operatorname{def}(a), \operatorname{def}(b)\},\{\operatorname{def}(c)\}\rangle$ & $\{c\}$ \\
\hline$W F S^{W K+L L C^{\prime}}\left(\Psi_{A F}\right)$ & $\langle\{\operatorname{def}(a), \operatorname{def}(b)\},\{\operatorname{def}(c)\}\rangle$ & $\{c\}$ \\
\hline$W F S^{+}\left(\Psi_{A F}\right)$ & $\langle\{\operatorname{def}(a), \operatorname{def}(b)\},\{\operatorname{def}(c)\}\rangle$ & $\{c\}$ \\
\hline
\end{tabular}

Even though in Example 2 all the argumentation semantics introduced in Lemma 2 were able to infer the same ideal set, one can identify different levels of inference with respect to each of those argumentation semantics. Let us consider the following example.

Example 3: Let $A F:=\langle A R$, attacks $\rangle$ be an argumentation framework, in which $A R:=\{a, b, c, d\}$ and attacks $:=$ $\{(a, b),(b, a),(a, c),(b, c),(c, d),(d, c)\}$ (see Figure 3).

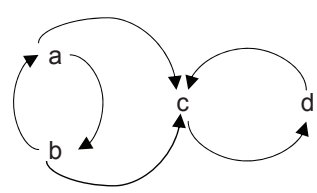

Figure 3. Graphic representation of $A F$ := $\langle\{a, b, c, d\},\{(a, b),(b, a),(a, c),(b, c),(c, d),(d, c)\}\rangle$.

One can see that the argumentation framework $A F$ has two preferred extensions: $\{a, d\}$ and $\{b, d\}$. On the other hand, we can also see that $A F$ has two ideal sets: \{\} and $\{d\}$. In the following table, we can see the different ideal sets inferred by the well-founded semantics and its extensions.

\begin{tabular}{|l|c|c|}
\hline Semantics & 3-logic programming model & Ideal Set \\
\hline$W F S\left(\Psi_{A F}\right)$ & $\langle\{\},\{\}\rangle$ & \{\} \\
\hline$W F S^{L L C^{\prime}}\left(\Psi_{A F}\right)$ & $\langle\{\},\{\}\rangle$, & \{\} \\
\hline$W F S^{W K}\left(\Psi_{A F}\right)$ & $\langle\{\operatorname{def}(c),\{\}\rangle$ & \{\} \\
\hline$W F S^{W K+L L C^{\prime}}\left(\Psi_{A F}\right)$ & $\langle\{\operatorname{def}(c)\},\{\}\rangle$ & \{\} \\
\hline$W F S^{+}\left(\Psi_{A F}\right)$ & $\langle\{\operatorname{def}(c)\},\{\operatorname{def}(d)\}\rangle$ & $\{d\}$ \\
\hline
\end{tabular}

As we can see, $W F S^{+}$is the only logic programming semantics which is able of inferring a non-empty ideal set.

In the following lemma, we define an order between the different ideal sets which were characterized in Lemma 2.

Lemma 3: Let $A F:=\langle A R$, attacks $\rangle$ be an argumentation framework. The following conditions hold:

$$
\begin{aligned}
\text { - } & I^{W F S}(A F) \subseteq I^{W F S^{L L C^{\prime}}}(A F), I^{W F S}(A F) \subseteq \\
& I^{W F S^{W K}}(A F) \\
\text { - } & I^{W F S^{L L C}}(A F) \quad \subseteq \quad I^{W F S^{W K+L L C^{\prime}}}(A F), \\
& I^{W F S^{W K}}(A F) \subseteq I^{W F S^{W K+L L C^{\prime}}}(A F) \\
\text { - } & I^{W F S^{W K+L L C^{\prime}}}(A F) \subseteq I^{W F S^{+}}(A F)
\end{aligned}
$$

Proof: (sketch) The lemma follows by the fact that these order relations are satisfied by $W F S, W F S^{L L C^{\prime}}$, $W F S^{W K}, W F S^{W K+L L C^{\prime}}$ and $W F S^{+}$.

Observe that the maximal ideal set that we have characterized in Lemma 2 is gotten by $W F S^{+}$.

Following the scepticism of the idea semantics from argumentation theory, we introduce the concept of idea model of a logic normal program. The construction of ideal models will be based on the p-stable semantics.

Definition 11: Let $P$ be a normal logic program and $M \subseteq$ $\mathcal{L}_{P} . M$ is an ideal model of $P$ if and only if $M$ is a model of $P$ and $\forall M^{\prime} \in P$ stable $(P), M^{\prime} \subseteq M$.

In order to illustrate this definition, let us consider the following example.

Example 4: Let $P$ be the following normal logic program:

$$
\begin{array}{ll}
\operatorname{def}(a) \leftarrow \text { not def }(b) . & \operatorname{def}(a) \leftarrow \operatorname{def}(a) . \\
\operatorname{def}(b) \leftarrow \text { not def }(a) . & \operatorname{def}(b) \leftarrow \operatorname{def}(b) .
\end{array}
$$

It is easy to see that $P$ has three 2-valued models: $S_{1}=$ $\{\operatorname{def}(a)\}, S_{2}=\{\operatorname{def}(b)\}$ and $S_{3}=\{\operatorname{def}(a), \operatorname{def}(b)\}$. From these models, one can see that only $S_{1}$ and $S_{2}$ are p-stable models. Observe that, both $S_{1}$ and $S_{2}$ are subsets of $S_{3}$. This means that $S_{3}$ is an ideal model.

An basic property of ideal models w.r.t. $\Psi_{A F}$ is that it always has at least an ideal model.

Lemma 4: Let $A F:=\langle A R$,attacks $\rangle$ be an argumentation framework. $\Psi_{A F}$ has always an ideal model which is $\mathcal{L}_{\Psi_{A F}}$

Proof: (sketch) Since every p-stable model $M$ is a subset of $\mathcal{L}_{\Psi_{A F}}$, the lemma follows by the fact that $\mathcal{L}_{\Psi_{A F}}$ always is a model of $\Psi_{A F}$.

An important property of the ideal models is that they are able to characterize ideal sets via the transformation function $\Psi$.

Theorem 3: Let $A F:=\langle A R$, attacks $\rangle$ be an argumentation framework. $S$ is an ideal model of $\Psi_{A F}$ if and only if $A R \backslash\{a \mid \operatorname{def}(a) \in S\}$ is an ideal set. 
Proof: (sketch) The theorem follows by the following observations:

- The 2-valued models of $\Psi_{A F}$ characterize the admissible sets of $A F$;

- The p-stable models of $\Psi_{A F}$ characterizes the preferred extensions of $A F$;

- Every ideal model contains every p-stable model of $\Psi_{A F}$.

Let us illustrate this theorem with the following example. Example 5: Let $A F:=\langle A R$, attacks $\rangle$ be an argumentation framework, where $\mathrm{AR}:=\{a, b\}$ and attacks := $\{(a, b),(b, a)\}$ (see Figure 4). $\Psi_{A F}$ corresponds to the program $P$ of Example 4 . We know that $\Psi_{A F}$ has only one ideal model which is: $\{\operatorname{def}(a), \operatorname{def}(b)\}$. Hence, $A F$ has only one ideal set which is: \{\} .

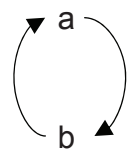

Figure 4. Graphic representation of $A F:=\langle\{a, b\},\{(a, b),(b, a)\}\rangle$.

\section{CONClusions AND Future WORK}

We showed that different extensions of the well-founded semantics can characterize different ideal sets (see Lemma 2). From all the ideal sets which were characterized in Lemma $2, I^{W F S^{+}}$is the maximal (w.r.t. set inclusion) ideal set which was characterized (see Lemma 3 ). We conjecture that $I^{W F S^{+}}$characterizes the maximal ideal set of an argumentation framework. Observe that if $I^{W F S^{+}}$characterizes the maximal ideal set of an argumentation framework, then our approach characterizes the semi-lattice of ideal sets of an argumentation framework. Part of our future work will be to prove these conjectures.

In order to capture the idea of ideal sets in logic programming, we have introduced the concept of ideal model. The construction of ideal models is based on p-stable models. We showed that ideal models define an interesting set of models which characterize the ideal sets of an argumentation framework in a direct way.

\section{ACKNOWLEDGMENT}

This work has been partially supported by the CONACyT [CB-2008-01 No.101581].

\section{REFERENCES}

[1] P. Baroni and M. Giacomin. Skepticism relations for comparing argumentation semantics. Int. J. Approx. Reasoning, 50(6):854-866, 2009.

[2] S. Brass, U. Zukowski, and B. Freitag. Transformationbased bottom-up computation of the well-founded model. In NMELP, pages 171-201, 1996.
[3] J. L. Carballido, J. C. Nieves, and M. Osorio. Inferring Preferred Extensions by Pstable Semantics. Iberoamerican Journal of Artificial Intelligence (Inteligencia Artificial) ISSN: 1137-3601, (doi: 10.4114/ia.v13i41.1029), 13(41):38$53,2009$.

[4] C. I. Chesñevar, A. G. Maguitman, and R. P. Loui. Logical models of argument. ACM Comput. Surv., 32(4):337-383, 2000 .

[5] J. Dix. A classification theory of semantics of normal logic programs: I. strong properties. Fundam. Inform., 22(3):227$255,1995$.

[6] J. Dix. A classification theory of semantics of normal logic programs: II. weak properties. Fundam. Inform., 22(3):257$288,1995$.

[7] J. Dix, M. Osorio, and C. Zepeda. A general theory of confluent rewriting systems for logic programming and its applications. Ann. Pure Appl. Logic, 108(1-3):153-188, 2001.

[8] P. M. Dung. On the acceptability of arguments and its fundamental role in nonmonotonic reasoning, logic programming and n-person games. Artificial Intelligence, 77(2):321-358, 1995.

[9] P. M. Dung, P. Mancarella, and F. Toni. Computing ideal sceptical argumentation. Artificial Intelligence, 171(issues 1015):642-674, 2007.

[10] P. E. Dunne. The computational complexity of ideal semantics. Artif. Intell., 173(18):1559-1591, 2009.

[11] U. Egly, S. A. Gaggl, and S. Woltran. Aspartix: Implementing argumentation frameworks using answer-set programmin. In M. G. de la Banda and E. Pontelli, editors, International Conference of Logic Programming (ICLP), volume 5366 of Lecture Notes of Computer Science, pages 734-738. Springer, 2008.

[12] A. J. García and G. R. Simari. Defeasible logic programming: An argumentative approach. Theory and Practice of Logic Programming, 4(1-2):95-138, 2004.

[13] A. V. Gelder, K. A. Ross, and J. S. Schlipf. The well-founded semantics for general logic programs. Journal of the ACM, 38(3):620-650, 1991.

[14] J. C. Nieves, M. Osorio, and U. Cortés. Preferred Extensions as Stable Models. Theory and Practice of Logic Programming, 8(4):527-543, July 2008.

[15] M. Osorio, J. A. Navarro, J. R. Arrazola, and V. Borja. Logics with Common Weak Completions. Journal of Logic and Computation, 16(6):867-890, 2006.

[16] T. Wakaki and K. Nitta. Computing Argumentatoin Semantics in Answer Set Progamming. In JSAI'2008, volume 5447 of Lecture Notes in Computer Science, pages 254-269, 2009. 